\title{
ON TWO TYPES OF EXOTIC ADDITION
}

\author{
SAM NorTHSHIELD \\ Department of Mathematics, SUNY-Plattsburgh \\ Plattsburgh, NY 12901
}

Abstract. We classify, under reasonable assumptions, all differentiable functions $f$ for which the 'secant method' $[x f(y)-y f(x)] /[f(y)-f(x)]$ is continuous and associative. Further, we classify all differentiable functions for which the similar type of addition $x f(y)+y f(x)$ is associative.

\section{INTRODUCTION.}

Let $\overline{\mathbb{R}}$ be the one-point compactification of $\mathbb{R}, f: \overline{\mathbb{R}} \rightarrow \overline{\mathbb{R}}$ and consider the binary operation

$$
x \oplus y=\frac{x f(y)-y f(x)}{f(y)-f(x)} .
$$

This operation is of course undefined when $x=y$ or when $f(x)=f(y)$. It is possible however to extend the definition of $\oplus$ for some but not all pairs of numbers $x, y$. We shall define a domain $D$ upon which an exteded definition is possible and upon which $\oplus$ is closed (i.e., $x \oplus y$ is defined and in $D$ for all $x, y \in D$ ) and continuous:

$$
\lim _{z \rightarrow y} x \oplus z=x \oplus y
$$

This also requires certain conditions on $f(x)$. We shall list them now:

$$
\text { No line crosses the graph } y=f(x) \text { at more than two points. }
$$

$$
f(x) \text { is differentiable on } \overline{\mathbb{R}} \text {. }
$$

In this context, differentiable shall mean that the difference quotient, for $x_{0} \in \mathbb{R}$ satisfies

$$
\lim _{x \rightarrow x_{0}} \frac{f(x)-f\left(x_{0}\right)}{x-x_{0}} \text { exists in } \overline{\mathbb{R}}
$$

and at $\infty$ there exists a tangent line: either there exists $L \in \mathbb{R}$ such that

$$
\lim _{x \rightarrow \infty}(f(x)-L x) \text { exists in } \mathbb{R}
$$

AMS 2000 Subject Classification: Primary 39B22 ; Secondary 39B12, 26A18.

Key words and phrases: Associativity, Secant Method, algebraic addition, Möbius transformation. 
in which case the tangent line has equation of the form $y=L x+b$ or

$$
\lim _{x \rightarrow \infty} \frac{f(x)}{x}=\infty
$$

in which case we write $L=\infty$ and the tangent line is, formally, $x=\infty$. We say $f(\infty)=0$ if $L=0$.

We now define a domain $D$ for $\oplus$ and define $x \oplus y$ for any $x, y \in D$ : let

$$
D:=\overline{\mathbb{R}}-Z(f)
$$

where $Z(f):=\{x: f(x)=0\}$ is the set of zeros of $f($ recall $f(\infty)=0$ if $L=0$ ) and

$$
x \oplus y:= \begin{cases}(x f(y)-y f(x)) /(f(y)-f(x)) & \text { if } x \neq y, f(x) \neq f(y), x \neq \infty \neq y \\ x-f(x) / f^{\prime}(x) & \text { if } x=y, f^{\prime}(x) \neq 0, x \neq \infty \neq y \\ x-f(x) / L & \text { if } x \neq \infty, y=\infty, L \neq \infty \\ x & \text { if } x \neq \infty, y=\infty, L=\infty \\ \infty & \text { otherwise. }\end{cases}
$$

Continuity of $\oplus$ forbids more than one zero in $D$. To see this, suppose $z_{1}, z_{2} \in$ $\partial Z(f)$. Since $f$ is continuous, $z_{1}, z_{2} \in Z(f)$ and $z_{i} \oplus x=z_{i}(\mathrm{i}=1,2)$ for all $x \notin Z(f)$. Hence

$$
z_{1}=\lim _{x \rightarrow z_{2}, x \notin Z(f)} z_{1} \oplus x=z_{1} \oplus z_{2} .
$$

Similarly, $z_{2}=z_{1} \oplus z_{2}$ and thus $z_{1}=z_{2}$. If there is one zero $z_{0} \in D$ then either $D-\left\{z_{0}\right\}$ is closed under $\oplus$ or not (e.g., if $f(x)=x^{2}$ or $f(x)=x$ respectively). In the first case, then we can proceed with $D-\left\{z_{0}\right\}$ instead of $D$. In the second case, there exist $x_{0}, y_{0} \in D-\left\{z_{0}\right\}$ such that $x_{0} \oplus y_{0}=z_{0}$. By continuity of $\oplus, x_{0} \oplus D:=\left\{x_{0} \oplus x: x \in D\right\}$ is connected and contains $z_{0}$. If $f(x)$ is not linear, then $x_{0} \oplus D$ contains an interval which contains $z_{0}$. Furthermore, if $\oplus$ is associative, then $y \in x_{0} \oplus D$ implies $y \oplus y_{0}=z_{0}$ and thus $(y, f(y))$ lies on the line through $\left(x_{0}, f\left(x_{0}\right)\right)$ and $\left(y_{0}, f\left(y_{0}\right)\right)$. It follows that $f(x)$ is linear on some interval containing $z_{0}$. We remark that this does not preclude non-trivial examples where this phenomenon could occur (the simplest such cases being where $f(x)$ is linear and the theory is trivial); rather we choose not to pursue this question at this time. It is worth noting that it is conceivable that rational functions may define an operation $\oplus$ via equation (1) on a suitably restricted domain. We do not address that question here either.

Note that condition (A) is equivalent to the reducibility of $\oplus$ :

$$
x \oplus y=x \oplus z \text { implies } y=z .
$$

At first glance, it looks that condition (A) is needlessly restrictive. Note, however, if $\oplus$ is associative and there exist three distinct points $(x, f(x)),(y, f(y))$, and $(z, f(z))$ all on a line, then $x \oplus y=x \oplus z=y \oplus z$. Let $u$ be the common value. By associativity, $u \oplus x=u \oplus y$ and thus the line through $(u, f(u))$ and $(x, f(x))$ coincides with the line through $(u, f(u))$ and $(y, f(y))$ It follows that $f(u)=0$ and thus $D$ is not closed. 
Condition (D) is necessary for $x \oplus x$ to be defined. To see this, note that $\oplus$ can be defined equivalently by

$$
x \oplus y=x-\frac{f(x)}{s(x, y)}
$$

where

$$
s(x, y):=\frac{f(x)-f(y)}{x-y}
$$

is the slope of the secant line through the points $(x, f(x))$ and $(y, f(y))$. Clearly, $x \oplus x$ is defined if and only if $s(x, y)$ has a limit as $y \rightarrow x$; i.e., $f$ is differentiable and we have

$$
x \oplus x=x-\frac{f(x)}{f^{\prime}(x)} \text { for } x \in \mathbb{R}-Z(f)
$$

with the understanding that

$$
x \oplus x=\infty \text { if } f^{\prime}(x)=0 .
$$

Equations (2a) and (2b) help to define addition involving infinity. If (2a) holds, then

$$
x \oplus \infty=x-\frac{f(x)}{L} \text { and } \infty \oplus \infty=\lim _{x \rightarrow \infty} x \oplus \infty .
$$

If $(2 \mathrm{~b})$ holds, then

$$
x \oplus \infty=x \text { and } \infty \oplus \infty=\infty .
$$

These considerations show that $x \oplus y$ is defined whenever $x \neq y$. Furthermore, $\oplus$ is closed (i.e., takes $D \times D$ to $D$ ) because if $x \neq y$ and $f(x \oplus y)=0$, then $(x, f(x))$, $(y, f(y))$, and $(x \oplus y, 0)$ are three distinct points on a line (violating assumption (A)). A similar argument holds for $x=y$.

We claim now that $s(x, y)$ is locally monotonic in each variable; we shall show this for $y>x$ and sufficiently close to $x$. Suppose $x<y<z<w$ for $y, z, w$ otherwise arbitrarily chosen from some interval on which $s(x, \cdot)$ is bounded. If $s(x, y)<s(x, z)>s(x, w)$, then let $L$ be a line through $(x, f(x))$ with slope between $s(x, z)$ and $\max \{s(x, y), s(x, w)\}$. Then $L$ divides the plane into two half-planes with $(z, f(z))$ in one and the points $(y, f(y))$ and $(w, f(w))$ in the other. By the intermediate value theorem, $L$ intersects the graph at $(x, f(x))$ and two other points as well - violating condition (A). A similar argument rules out $s(x, y)>s(x, z)<s(x, w)$ and so $s(x, \cdot)$ is monotonic on some interval $(x, x+\epsilon)$.

Suppose $f(x \oplus x)=0$. Then the tangent line through $(x, f(x))$ goes through $(x \oplus x, 0)$. Since, by $(\mathrm{A}), f^{\prime}(x)$ is locally monotonic, the curve $y=f(x)$ is locally either below or above the tangent line at $(x, f(x))$ and so a line through $(x \oplus x, 0)$ with slope slightly lower or higher than $f^{\prime}(x)$ must intersect the curve at more than two places (in violation of (A)). Hence $x \oplus x \in D$.

The binary operation $\oplus$ is, as mentioned before, closely related to the secant method and Newton's method. Given two initial distinct approximations $x_{0}$ and $x_{1}$ to a root of $f(x)=0$, we get a new approximation $x_{2}:=x_{0} \oplus x_{1}$. Repeating this process to initial guesses $x_{1}$ and $x_{2}$ gives $x_{3}$ and so on. The secant method is the computation of the sequence $\left(x_{n}\right)$ where

$$
x_{n+1}=x_{n} \oplus x_{n-1} .
$$


Newton's method is the iteration of the map

$$
x \mapsto x \oplus x=x-\frac{f(x)}{f^{\prime}(x)} .
$$

For certain functions $f, \oplus$ is associative; when this is so, we say that $\oplus$ is a secant addition and the sequence $\left(x_{n}\right)$ of approximations from the secant method becomes

$$
x_{n}=\overbrace{x_{0} \oplus \cdots \oplus x_{0}}^{F_{n-1} \text { times }} \oplus \overbrace{x_{1} \oplus \cdots \oplus x_{1}}^{F_{n} \text { times }}
$$

where $\left(F_{n}\right)$ is the Fibonacci sequence. Furthermore, a function $G$ satisfying

$$
G(x \oplus y)=G(x)+G(y)
$$

exists and can always be found and so, a closed formula for $x_{n}$ can be found when $G$ is invertible:

$$
x_{n}=G^{-1}\left(F_{n-1} \cdot G\left(x_{0}\right)+F_{n} \cdot G\left(x_{1}\right)\right) .
$$

Several addition laws which appear in physics and mathematics are actually secant additions. For $f(x)=\left(c^{2}-x^{2}\right) / x$, the induced secant addition is

$$
x \oplus y=\frac{x+y}{1+x y / c^{2}}
$$

which is the law of addition of velocities in special relativity. For $f(x)=x^{2}$, the induced secant addition is

$$
x \oplus y=\frac{1}{1 / x+1 / y}
$$

which is law of addition of parallel resistances. For $f(x)=1 / x$ and $f(x)=x /(1-$ $x)$, the induced secant additions are normal addition and normal multiplication respectively.

The secant method can be thought of as a geometric way of combining two points in part of a curve to get a third point on the curve. If associative, such a method is called a "group law" and it is known that certain curves always possess a group law. For example by the Cayley-Bacharach Theorem (see, for example, the book by Silverman and Tate $[\mathrm{ST}]$ ), cubic curves have an associative group law and, in particular, the curves

$$
y^{2}(d x+e)=y\left(a x^{2}+b x+c\right)
$$

have one. For these curves, the geometric addition prescribed by the CayleyBacharach Theorem actually coincides with the secant method for functions of the form

$$
f(x)=\frac{a x^{2}+b x+c}{d x+e} .
$$

Therefore, for all functions of the form (5), the binary operation (1) is associative. A proof of this fact appears in [N2] and uses Pascal's theorem of 1640 of which the Cayley-Bacharach theorem is a modern generalization. For completeness, we present the proof here. 
Proposition 1. If $f(x)=\left(a x^{2}+b x+c\right) /(d x+e)$ where $a$ and $d$ are not both 0 then $\oplus$ is a secant addition.

Proof. Pascal's theorem states that the points of intersection of opposite sides of a hexagon inscribed in a conic all lie on a common line. Specifically, If $\bar{x}_{1}, \bar{x}_{2}, \bar{x}_{3}, \bar{x}_{4}, \bar{x}_{5}$, and $\bar{x}_{6}$ are six distinct points on a conic section, then the points of intersection $L\left(\bar{x}_{1}, \bar{x}_{2}\right) \cap L\left(\bar{x}_{4}, \bar{x}_{5}\right), L\left(\bar{x}_{2}, \bar{x}_{3}\right) \cap L\left(\bar{x}_{5}, \bar{x}_{6}\right)$, and $L\left(\bar{x}_{3}, \bar{x}_{4}\right) \cap L\left(\bar{x}_{6}, \bar{x}_{1}\right)$ (where $L(\bar{x}, \bar{y})$ denotes the line through the points $\bar{x}$ and $\bar{y}$ ) all lie on a straight line.

Given real $x$, let $\bar{x}$ denote the point $(x, f(x))$. Given distinct real numbers $x, y$ and $z$ on the x-axis such that $x \oplus y \neq z$ and $x \neq y \oplus z$, consider the sequence of six points on the conic section $y=f(x): \bar{x}, \bar{y}, \bar{z}, \overline{x \oplus y}, \infty$, and $\overline{y \oplus z}$. Note that the lines $L(\bar{x}, \bar{y})$ and $L(\overline{x \oplus y}, \infty)$ intersect on the x-axis at the point corresponding to $x \oplus y$. Replacing $x$ by $z$, we get a similar intersection at $z \oplus y$ and so, by Pascal's theorem, the lines $L(\bar{x}, \overline{y \oplus z})$ and $L(\overline{x \oplus y}, \bar{z})$ intersect on the x-axis. By definition of $\oplus$, the first line intersects the $\mathrm{x}$-axis at $x \oplus(y \oplus z)$ while the second line intersects the $\mathrm{x}$-axis at $(x \oplus y) \oplus z$ and so the quantities $(x \oplus y) \oplus z$ and $x \oplus(y \oplus z)$ are equal. This shows that $\oplus$ is weakly associative: i.e., associativity holds for triples avoiding confluence. Associativity itself follows from differentiability (and continuity) of $f(x)$.

As an application, we shall analyze iterates of the Möbius transformation $m(x):=(a x+b) /(c x+d)$. Given $x_{0} \in \overline{\mathbb{R}}$, define $\left(x_{n}\right)$ recursively by $x_{n+1}=m\left(x_{n}\right)$. Let $e:=m^{-1}\left(x_{0}\right)$ and let $f(x)=\frac{c x^{2}+(d-a) x-b}{x-e}$. Note that the numerator is a characteristic polynomial of $m$ (i.e., the polynomial whose roots are the fixed points of $m$ ). Let $\oplus$ denote the secant addition induced by $f$. A direct calculation gives

$$
x \oplus y=\frac{(a-d-c e) x y+b(x+y)-e b}{c x y-c e(x+y)+(a-d) e+b} .
$$

Then $x \oplus m(e)$ is a Möbius transformation and $e \oplus x=x$ for all $x$. It turns out that

$$
m(x)=x \oplus m(e)=x \oplus x_{0} .
$$

This is easily seen by noting that the Möbius transformations $m(x)$ and $x \oplus m(e)$ have the same characteristic polynomials $(m(e) \oplus x=x$ iff $f(x)=0$ iff $p(x)=0)$ and agree at some value other than a fixed point $(m(e)=e \oplus m(e))$.

By associativity, the n-fold 'sum' $c \oplus c \oplus \cdots \oplus c$ is well defined and we shall denote it by $c^{\oplus n}$. Therefore,

$$
m_{n}\left(x_{0}\right)=x_{0}^{\oplus n}
$$

The Newton-Raphson method (or Newton's method, see [BB]) applied to the function $f$ at $x$ gives $x \oplus x$. Hence, the n-th iterate of the Newton-Raphson method, starting at $x_{0}$, gives $x_{0}^{\oplus 2^{n}}=m_{2^{n}}\left(x_{0}\right)$.

A further application is a short proof of 'Aitken acceleration'; see [N1]. Let $x_{n}$ be a sequence defined by

$$
x_{n+1}=a x_{n}+b x_{n-1}
$$


and define

$$
r_{n}=\frac{x_{n+1}}{x_{n}}
$$

Then $r_{n+1}=a+b / r_{n}$ and so

$$
r_{n}=m_{n}\left(r_{0}\right)
$$

the n-th iterate of the Möbius transformation $m(x)=a+b / x$. If $\oplus$ is the secant addition corresponding to the characteristic polynomial $f(x)=x^{2}-a x-b$, i.e.

$$
x \oplus y:=\frac{x y+b}{x+y-a}
$$

then $r_{n+1}=r_{n} \oplus a$ and therefore $r_{n}=r_{0} \oplus a^{\oplus n}$ and, for all $n, k \geq 0$,

$$
r_{n+k}=r_{k} \oplus a^{\oplus n}
$$

Then

$$
\frac{r_{n}^{2}+b}{2 r_{n}-a}=r_{n} \oplus r_{n}=r_{0} \oplus r_{0} \oplus a^{\oplus 2 n}=\frac{r_{n+k} r_{n-k}+b}{r_{n+k}+r_{n-k}-a}
$$

and, since they are equal, they are also equal to the ratio of their differences:

$$
r_{0} \oplus r_{2 n}=\frac{r_{n+k} r_{n-k}-r_{n}^{2}}{r_{n+k}-2 r_{n}+r_{n-k}} .
$$

Other consequences of associativity appear in [N1] and [N2].

It is not true that $\oplus$ is associative for all functions $f$. For example, if $f(x)=x^{3}$, then $x \oplus y=\left(x^{2} y+x y^{2}\right) /\left(x^{2}+x y+y^{2}\right)$ for $x \neq y$ and $x \oplus x=2 x / 3$ which is not associative $[(1 \oplus 1) \oplus-1=2 / 7 \neq 0=1 \oplus(1 \oplus-1)]$. The question we ask now is "are there any secant additions other than those induced by functions of the form $\left(a x^{2}+b x+c\right) /(d x+e)$ ?" The answer is, generally, 'no'.

Theorem 1. For $f: \overline{\mathbb{R}} \rightarrow \overline{\mathbb{R}}$ satisfying conditions $(A)$ and $(D), \oplus$ is associative if and only if $f$ is of the form $f(x)=\frac{a x^{2}+b x+c}{d x+e}$ where a and $d$ are not both 0 .

This result contains a partial converse of Pascal's theorem in the sense that any graph of a function for which Pascal's theorem holds must be a conic section. We shall prove Theorem 1 in Section 2.

Secant addition turns out to arise via matrices. Suppose $A$ is a two-by-two matrix. Since $A^{2}$ is a linear combination of $A$ and $I$ (by the Cayley-Hamilton theorem), then either, for some $c$,

$$
(A-x I)(A-y I)=c I
$$

or there exist unique numbers $k$ and $z$ such that

$$
(A-x I)(A-y I)=k(A-z I) .
$$


To see this, suppose $A^{2}=a A+b I$. If $x+y=a$, then the first case holds with $c=x y+b$ while if $x+y \neq a$, then the second case holds with $k=a-x-y$ and $z=(x y+b) /(x+y-a)$.

Then $z$ depends on $x$ and $y$ and we shall define a type of addition $\oplus$ by $x \oplus y:=z$. We let $\doteq$ denote projective equality: $A \doteq B$ if and only if $A=k B$ for some scalar $k$, and thus

$$
(A-x I)(A-y I) \doteq A-(x \oplus y) I .
$$

The operation $\oplus$ inherits associativity from matrix multiplication:

$$
A-(x \oplus(y \oplus z)) I \doteq(A-x I)(A-y I)(A-z I) \doteq A-((x \oplus y) \oplus z) I .
$$

It will be shown (Theorem 2 ) that $\oplus$ is secant addition induced by the characteristic polynomial $f$ of $A$ and, in fact, all secant additions can be interpreted in this way.

Theorem 2. Suppose $\oplus$ is an associative, and commutative binary operation. The following are equivalent:

a) $\oplus$ is of secant type, i.e.,

$$
x \oplus y=\frac{x f(y)-y f(x)}{f(y)-f(x)}
$$

for some function $f$ satisfying conditions $(A)$ and $(D)$

b) $\oplus$ is of secant type with respect to a function $f$ of the form

$$
f(x)=\frac{a x^{2}+b x+c}{d x-e}
$$

where $a$ and $d$ are not both 0 ,

c) $\frac{\partial}{\partial x}(x \oplus y)=\frac{p(x \oplus y)}{p(x)}$ for some polynomial $p$ of degree at most 2,

d) there exists $e \in \overline{\mathbb{R}}$ such that $x \oplus e=x$ and, for all $c \in \mathbb{R}$ the function $m(x):=$ $x \oplus c$ is either a Möbius transformation or is constant,

e) there exists a two-by-two real matrix $A$ and $e \in \overline{\mathbb{R}}$ not in the spectrum of $A$ such that

$$
(A-x I)(A-y I) \doteq(A-(x \oplus y) I)(A-e I)
$$

where $M \doteq N$ means $M=c N$ for some $c \in \mathbb{R}($ and $A-\infty I=-I)$.

Secant addition also arises in the theory of differential equations. For example, $y:=\cos x$ is a solution of $y^{\prime \prime}+y=0$ and $-y^{\prime} / y(=\tan x)$ satisfies

$$
\tan (x+y)=\tan (x) \oplus \tan (y)
$$

where

$$
u \oplus v:=\frac{u+v}{1-u v}
$$

is the secant addition induced by the characteristic function $x^{2}+1$. This can be broadly generalized. 
Theorem 3. If $y$ is a solution of $y^{\prime \prime}-\alpha y^{\prime}+\beta y=0$ and $T(x):=-y^{\prime}(x) / y(x)$ then $T$ satisfies the functional equation

$$
T(x+y)=T(x) \oplus T(y)
$$

where $\oplus$ is the secant addition (1) generated by the function

$$
f(x)=\frac{x^{2}+\alpha x+\beta}{x-T(0)}
$$

if $T(0) \neq \infty$ (equivalently, $y(0) \neq 0$ ) or generated by

$$
f(x)=x^{2}+\alpha x+\beta
$$

if $T(0)=\infty$ (equivalently, $y(0)=0)$.

Hence, by Theorem 1, all secant additions arise in this way.

We next consider another class of binary operations. For example, consider

$$
x \diamond y:=x \sqrt{1-y^{2}}+y \sqrt{1-x^{2}} \text { for } x, y \in[-1,1] .
$$

It turns out to be associative since the sine function acts as a homomorphism:

$$
\sin (x+y)=\sin (x) \diamond \sin (y) \text { for } x \in\left[\frac{-\pi}{2}, \frac{\pi}{2}\right] .
$$

For this reason, we say that any associative binary operation defined by

$$
x \diamond y=x \varphi(y)+y \varphi(x)
$$

for some differentiable function $\varphi$ is a sine-type addition.

It turns out that sine-type additions always arise from differential equations.

Corollary 1. If $y^{\prime \prime}-\alpha y^{\prime}+\beta y=0, y(0)=0$, and $y^{\prime}(0)=1$, and if $\varphi \circ y=y^{\prime}-\frac{1}{2} \alpha y$, then the addition defined by (6) is associative. Furthermore, every sine-type addition arises this way.

This is a corollary of the following theorem:

Theorem 4. Suppose $\diamond$ is a binary operation of the form

$$
x \diamond y=x \varphi(y)+y \varphi(x) \text { for } x, y \in I
$$

where $I$ is an interval containing $0, \varphi$ is differentiable, $a:=\varphi^{\prime}(0), \varphi(0)=1$, and

$$
s^{-1}(x)=\int_{0}^{x} \frac{d t}{\varphi(t)+a t} .
$$


The following are equivalent:

a) $\diamond$ is associative,

b) $\varphi(x \diamond y)=\varphi(x) \varphi(y)+k x y$ for some $k \in \mathbb{R}$,

c) $\varphi^{\prime}(x \diamond y)=\frac{\varphi^{\prime}(x) \varphi(y)+k y}{\varphi(y)+y \varphi^{\prime}(x)}$,

d) $\varphi^{\prime}(x)=\frac{a \varphi(x)+k x}{\varphi(x)+a x}$

e) $s^{\prime \prime}-2 a s^{\prime}+\left(a^{2}-k\right) s=0, s(0)=0, s^{\prime}(0)=1$,

f)

$$
s(x)=\left\{\begin{array}{l}
\frac{1}{\sqrt{k}} e^{a x} \sinh (\sqrt{k} x) \text { if } k>0, \\
\frac{1}{\sqrt{-k}} e^{a x} \sin (\sqrt{-k} x) \text { if } k<0, \\
x e^{a x} \text { if } k=0 .
\end{array}\right.
$$

g) $s(x+y)=s(x) \diamond s(y)$,

h) $s^{-1}(x \diamond y)=s^{-1}(x)+s^{-1}(y)$,

i) $\frac{\partial}{\partial x}(x \diamond y)=\frac{\varphi(x \diamond y)+a \cdot(x \diamond y)}{\varphi(x)+a x}$.

Suppose $\diamond$ is a sine-type addition induced by the function $\varphi$ and let $\Phi(x):=$ $\varphi(x) / x$. By the equivalence of (a) and (b) in Theorem 4,

$$
\Phi(x \diamond y)=\Phi(x) \oplus \Phi(y)
$$

where $\oplus$ is the secant addition induced by $f(x)=x^{2}-k$ :

$$
x \oplus y=\frac{x y+k}{x+y} .
$$

The solution of the equation $g(x)+g(y)=h(x f(y)+y f(x))$ for all three unknown functions $f, g$ and $h$ was accomplished by Abel in 1827 and uses the idea of reducing to differential equations. Cayley also considered this equation; see $[A 1$, p.8] for references. For continuous solutions (i.e., partial solution of the second part of Hilbert's fifth problem), see the several papers by Sablik [S1,S2] and a paper by Aczel [A2]. See also the comments after Lemma 4 below.

The theory covered in this paper may also be done over the Riemann sphere $\overline{\mathbb{C}}$; several of our arguments, however, depend on the order properties of the real line and do not carry over verbatim to the complex case.

Another natural setting for this theory is projective space (either real or complex). To some extent, this type of thing has been done; we now present some connections between our work and known results. Define $\varphi: D \rightarrow \mathbb{R}^{2}$ by

$$
\varphi(x)=\left(\frac{1}{f(x)}, \frac{x}{f(x)}\right)
$$

with special cases $\varphi(\infty):=(0,1 / L)$ if $\lim _{x \rightarrow \infty} f(x) / x=L$ and $\varphi(e)=(0,0)$ if $f(e)=\infty$. 
Let $\mathcal{C}:=\varphi(D)$. Note that $\mathcal{C}$ inherits property (A); that is, no three points $\varphi(x), \varphi(y), \varphi(z)$ lie on a straight line for, if not, there exist numbers $x, y, z$, and $t$ such that

$$
(1-t)\left(\frac{1}{f(x)}, \frac{x}{f(x)}\right)+t\left(\frac{1}{f(y)}, \frac{y}{f(y)}\right)=\left(\frac{1}{f(z)}, \frac{z}{f(z)}\right)
$$

which, for $a:=(1-t) f(z) / f(x)$ and $b:=t f(z) / f(y)$, implies

$$
a(x, f(x))+b(y, f(y))=(z, f(z))
$$

By Lemma $1, \mathbf{0}:=(0,0) \in \mathcal{C}$. We introduce an equivalence relation on $\mathbb{R}^{2}$ :

$$
(x, y) \doteq(u, v) \text { if there exists } k \in \mathbb{R}-\{0\} \text { such that } x=k u \text { and } y=k v \text {. }
$$

We may then define a binary operation '*' on $\mathcal{C}$ : given $u, v \in \mathcal{C}$, let $u * v$ be the unique element in $\mathcal{C}$ such that $u * v \doteq u-v$. Geometrically, $u * v$ is that point on $\mathcal{C}$ such that the line through it and $\mathbf{0}$ is parallel to the line through $u$ and $v$.

Proposition 2. For $x, y \in D$,

$$
\varphi(x) * \varphi(y)=\varphi(x \oplus y) .
$$

Proof. $\varphi(x \oplus y) \doteq(1, x \oplus y) \doteq(f(y)-f(x), x f(y)-y f(x))$

$$
\doteq\left(\frac{1}{f(x)}-\frac{1}{f(y)}, \frac{x}{f(x)}-\frac{y}{f(y)}\right)=\varphi(x)-\varphi(y) \doteq \varphi(x) * \varphi(y) \text {. }
$$

Hence $*$ and $\oplus$ are conjugate. In particular, the associativity of $\oplus$ is equivalent the associativity of $*$. We note other properties equivalent to the associativity of *. We define a binary relation $\sim$ on $\mathcal{C} \times \mathcal{C}$ :

$$
(u, v) \sim(w, z) \text { iff } z-u \doteq w-v \text { iff } z * u=w * v
$$

This equivalence relation appears (we correct a misprint) in problem 21 of the ISFE \#9 meeting website $[\mathrm{Au}]$ where it is stated that this is equivalent to $\mathcal{C}$ being a conic (under certain additional conditions).

Given two points $A, B$ in the plane, let $\overline{A B}$ denote the line through $A$ and $B$ and, for lines $L_{1}$ and $L_{2}$, let $L_{1} \| L_{2}$ mean that $L_{1}$ and $L_{2}$ are parallel. We now define the Parallel Hexagon Property (PHP) for a curve $\mathcal{C}$ : if $A, B, C, D, E, F$ are six points in $\mathcal{C}$, and if $\overline{A B} \| \overline{D E}$ and $\overline{B C} \| \overline{E F}$ then $\overline{C D} \| \overline{F A}$. This property is possessed by all conics in that it is a special case of Pascal's theorem (i.e., two pairs of opposite sides of an inscribed hexagon cross at infinity implies that the third pair does as well - see Proposition 1).

Proposition 3. The transitivity of $\sim$ is equivalent to the associativity of $*$ which is equivalent to the PHP.

Proof. Assume the associativity of $*$ and suppose $(a, b) \sim(u, v)$ and $(u, v) \sim$ $(c, d)$. That is, $a * v=b * u$ and $u * d=v * c$. Then $a * v * d=b * u * d=b * v * c$ 
and, by the reducibility of $*, a * d=b * c$ and thus $(a, b) \sim(c, d)$. Therefore $\sim$ is transitive.

Suppose now that $\sim$ is transitive. Let $A, B, C, D, E, F \in \mathcal{C}$ where $\overline{A B} \| \overline{D E}$ and $\overline{B C} \| \overline{E F}$. The first shows that $A-B \doteq D-E$ and thus $(A, D) \sim(B, E)$ and, similarly, the second shows that $(B, E) \sim(C, F)$. By transitivity, $(A, D) \sim(C, F)$ and thus $\overline{C D} \| \overline{F A}$ and the PHP holds.

Supposing the PHP, let $u, v, w \in \mathcal{C}$ and consider the sequence $\mathbf{0}, v * w, u, v, w, u *$ $v$. Since, by definition $\overline{\mathbf{0}(u * v)}|| \overline{u v}$ and $\overline{\mathbf{0}(v * w)} \| \overline{v w}$, we have by the PHP,

$$
\overline{u(v * w)}|| \overline{(u * v) w} .
$$

That is, $u *(v * w)=(u * v) * w$ and thus $*$ is associative.

We sketch why associativity of $\oplus$ (and thus of $*$ ) implies that $\mathcal{C}$ is a conic. Let $\mathcal{C}_{0}$ be a segment of the curve $\mathcal{C}$ which is the graph of some function $g$ over some interval $I$. We may define the $g$-differential mean value on $\mathcal{C}_{0}(\mathrm{cf}[\mathrm{K}],[\mathrm{Bo}],[\mathrm{A} 3])$

$$
D_{g}(x, y):=\left(g^{\prime}\right)^{-1}\left(\frac{g(x)-g(y)}{x-y}\right) \text {. }
$$

Aumann showed (see $[\mathrm{K}],[\mathrm{A} 3]$ and references therein) that $\mathcal{C}_{0}$ is part of a conic section if $D_{g}$ is a quasi-arithmetic mean: i.e. if there exists a function $F$ such that

$$
D_{g}(x, y)=F^{-1}\left(\frac{F(x)+F(y)}{2}\right) .
$$

By [A1, p. 281], this occurs if $D_{g}$ is bisymmetric; if we define '.' on $\mathcal{C}_{0}$ by $u \cdot v$ being the unique point in $\mathcal{C}_{0}$ with tangent line parallel to the line through $u$ and $v$, then bisymmetry of $D_{g}$ translates to the bisymmetry of $\cdot$ :

$$
(a \cdot b) \cdot(c \cdot d)=(a \cdot c) \cdot(b \cdot d) .
$$

This follows from the associativity of $* *$ since

$$
a * b=(a \cdot b) *(a \cdot b)
$$

and thus

$$
((a \cdot b) \cdot(c \cdot d))^{* 4}=((a \cdot b) *(c \cdot d))^{* 2}=a * b * c * d .
$$

We say that Pascal's Property holds if the conclusion of Pascal's theorem holds (see Proposition 1).

Proposition 4. The following are equivalent: Pascal's Property holds, the Parallel Hexagon Property holds, $\oplus$ is associative, ${ }^{*}$ is associative, $\sim$ is an equivalence relation, · is bisymmetric, $D_{g}$ is quasi-arithmetic, $\mathcal{C}$ is a conic section.

The operation $\oplus$ is, under our assumptions, a group law. It turns out that all rational formal group laws have been classified by Coleman and McGuinness [CMc]. 
The author thanks the referees for many suggestions and references.

\section{PROOF OF THEOREM 1.}

Let $f(x)$ satisfy conditions (A) and (D) and suppose $\oplus$ is associative. In view of Proposition 1, to prove Theorem 1 it is enough to show that $f(x)$ is of the form $\left(a x^{2}+b x+c\right) /(d x+e)$ where $a$ and $d$ are not both 0 .

Recall $s(x, y):=(f(x)-f(y)) /(x-y)$ is the slope of the secant line through the points on the graph of $f$ corresponding to $x$ and $y$.

Lemma 1. $s(x, y)$ is unbounded. Consequently, $f(e)=\infty$ for some (unique) $e \in \mathbb{R}$ or $\lim _{x \rightarrow \infty} f(x) / x=\infty$.

Proof. Suppose that $s(x, y)$ is bounded. Equation (2b) is ruled out and so (2a) holds: for some $L \in \mathbb{R}$,

$$
\lim _{x \rightarrow \infty}(f(x)-L x) \text { exists in } \mathbb{R} .
$$

Fix $x_{0}$. As $x \rightarrow \infty$ (from both positive and negative directions),

$$
s\left(x_{0}, x\right)=\frac{f(x)-f\left(x_{0}\right)}{x-x_{0}}=\frac{f(x)-L x+L\left(x-x_{0}\right)+L x_{0}-f\left(x_{0}\right)}{x-x_{0}} \rightarrow L .
$$

Note that $s\left(x_{0}, \cdot\right)$ is continuous with a removable singularity at $x_{0}$ (in particular, $\left.s\left(x_{0}, x_{0}\right)=f^{\prime}\left(x_{0}\right)\right)$. If $f^{\prime}\left(x_{0}\right) \neq L$ then any line through $\left(x_{0}, f\left(x_{0}\right)\right)$ with slope between $L$ and $f^{\prime}\left(x_{0}\right)$ must intersect the graph of $f(x)$ at three or more points (contradicting $(\mathrm{A}))$. Hence $f^{\prime}(x)$ is constant, again contradicting $(\mathrm{A})$.

By Lemma 1, we may define a function:

$$
p(x):= \begin{cases}f(x), & \text { if } \lim _{x \rightarrow \infty} f(x) / x=\infty \\ f(x)(x-e), & \text { if } f(e)=\infty\end{cases}
$$

Lemma 2. $\frac{\partial}{\partial x}(x \oplus y)=\frac{p(x \oplus y)}{p(x)}$.

Proof. Note $s(x, y)=\frac{f(x)}{x-x \oplus y}$. Let $e^{\prime}$ be $e$ if $f(e)=\infty$, otherwise let $e^{\prime}=\infty$. In either case, $\lim _{z \rightarrow e^{\prime}} x \oplus z=x$. Then, by associativity, $x \oplus y \oplus z$ is well defined and

$\frac{\partial}{\partial x}(x \oplus y)=\lim _{z \rightarrow e^{\prime}} \frac{x \oplus y-x \oplus y \oplus z}{x-x \oplus z}=\lim _{z \rightarrow e^{\prime}} \frac{f(x \oplus y)}{s(x \oplus y, z)} \frac{s(x, z)}{f(x)}=\frac{f(x \oplus y)}{f(x)} \lim _{z \rightarrow e^{\prime}} \frac{s(x, z)}{s(x \oplus y, z)}$.

Note that

$$
\lim _{z \rightarrow e^{\prime}} \frac{s(x, z)}{s(x \oplus y, z)}=\lim _{z \rightarrow e^{\prime}} \frac{f(x)-f(z)}{x-z} \frac{x \oplus y-z}{f(x \oplus y)-f(z)}=\lim _{z \rightarrow e^{\prime}} \frac{x \oplus y-z}{x-z}
$$


which equals 1 if $e^{\prime}=\infty$ and equals $\frac{x \oplus y-e}{x-e}$ if $e^{\prime}=e$

The following Lemma is a well-known characterization of polynomials of degree at most two. It perhaps goes back to Lagrange.

Lemma 3. If for all $x, y(x \neq y)$ in an interval,

$$
\frac{g^{\prime}(x)+g^{\prime}(y)}{2}=\frac{g(x)-g(y)}{x-y},
$$

then $g$ is a polynomial of degree at most two.

Proof. Observe that the hypothesis implies that $g(x)$ is twice differentiable: fix $y \neq x$ and note that since the right side is differentiable at $x$, then so is the left. Equating the partial derivatives (with respect to $x$ ) of each side, we find

$$
g^{\prime \prime}(x) / 2=\frac{g^{\prime}(x)-(g(x)-g(y)) /(x-y)}{x-y}=\frac{g^{\prime}(x)-g^{\prime}(y)}{2(x-y)}
$$

and thus $g^{\prime \prime}(x)=g^{\prime \prime}(y)$. Hence $g^{\prime \prime}$ is constant.

Proof of Theorem 1. It is easy to verify that

$$
\frac{\partial}{\partial x} s(x, y)=\frac{f^{\prime}(x)-s(x, y)}{x-y}
$$

Since, by (3),

$$
x \oplus y=y-\frac{f(y)}{s(x, y)},
$$

we find

$$
\frac{\partial}{\partial x}(x \oplus y)=\frac{f(y)}{s(x, y)^{2}} \cdot \frac{f^{\prime}(x)-s(x, y)}{x-y} .
$$

By Lemma 2, there are two cases. If

$$
\frac{\partial}{\partial x}(x \oplus y)=\frac{f(x \oplus y)}{f(x)},
$$

then, using equation (7), we may write

$$
\frac{f(x \oplus y)}{f(x) f(y)} s(x, y)^{2}=\frac{f^{\prime}(x)-s(x, y)}{x-y} .
$$

Since the left side is symmetric in $x$ and $y$, so is the right side and we find

$$
f^{\prime}(x)-s(x, y)=s(x, y)-f^{\prime}(y)
$$

or, equivalently,

$$
\frac{f^{\prime}(x)+f^{\prime}(y)}{2}=\frac{f(x)-f(y)}{x-y} .
$$


By Lemma $3, f(x)=a x^{2}+b x+c$ for some $a, b, c$.

The other case is when

$$
\frac{\partial}{\partial x}(x \oplus y)=\frac{f(x \oplus y)(x \oplus y-e)}{f(x)(x-e)} .
$$

Using equation (7) here, we may write

$$
\frac{f(x \oplus y)(x \oplus y-e)}{f(x) f(y)} s(x, y)^{2}=\frac{(x-e)\left(f^{\prime}(x)-s(x, y)\right)}{x-y} .
$$

Since the left side is symmetric in $x$ and $y$, so is the right side and we find

$$
(x-e)\left(f^{\prime}(x)-s(x, y)\right)=(y-e)\left(s(x, y)-f^{\prime}(y)\right)
$$

or, equivalently,

$$
\frac{(x-e) f^{\prime}(x)+(y-e) f^{\prime}(y)}{x+y-2 e}=\frac{f(x)-f(y)}{x-y} .
$$

Letting $q(x):=x f(x+e), u=x-e$, and $v=y-e$, we may rewrite this as

$$
\frac{q^{\prime}(u)+q^{\prime}(v)}{2}=\frac{q(u)-q(v)}{u-v},
$$

and therefore, by Lemma $3, q(u)=a u^{2}+b u+c$ for some $a, b, c$. Then

$$
f(x)=\frac{q(x-e)}{x-e}=\frac{a x^{2}+(b-2 a e) x+\left(a e^{2}-b e+c\right)}{x-e} .
$$

\section{PROOF OF THEOREMS 2 AND 3.}

Proof of Theorem 2. $(a \Leftarrow \Rightarrow b)$ : This is Theorem 1 .

$(b \Rightarrow c)$ : If (b) holds, then $s(x, y)$ is unbounded. By Lemma 2, (c) holds.

$(c \Rightarrow d):$ The Schwarzian derivative

$$
S(m)=\left(m^{\prime \prime} / m^{\prime}\right)^{\prime}-\frac{1}{2}\left(m^{\prime \prime} / m^{\prime}\right)^{2}
$$

is zero if and only if $m$ is a Möbius transformation. Let $m(x)=x \oplus k$. By Lemma 2 , since $m^{\prime}(x)=\frac{\partial}{\partial x} x \oplus k=p(x \oplus k) / p(x)$,

$$
m^{\prime}=\frac{p \circ m}{p} .
$$

Using this to work out its Schwarzian derivative, $S(m)=(q \circ m-q) / p^{2}$ where $q=p p^{\prime \prime}-\frac{1}{2}\left(p^{\prime}\right)^{2}$. Since $p$ is a polynomial of degree at most $2, q$ is constant and so $S(m)=0$. 
$(d \Rightarrow e)$ : Given a matrix $M:=\left(\begin{array}{ll}a & b \\ c & d\end{array}\right)$, we define the corresponding Möbius function

$$
\Phi_{M}(x):=\frac{a x+b}{c x+d}
$$

By (d), there exist matrices $A(x)$ such that

$$
x \oplus y=\Phi_{A(x)}(y) .
$$

Hence, for $x, y, z \in \mathbb{R}$,

$$
\Phi_{A(x) A(z)}(y)=\Phi_{A(x)}\left(\Phi_{A(z)}(y)\right)=x \oplus(y \oplus z)
$$

and thus the matrices $A(x)$ all commute (modulo the equivalence relation $\doteq$ ). Furthermore, for all $x, y$,

$$
A(x \oplus y) \doteq A(x) A(y)
$$

Fix $k \in \mathbb{R}$ such that

$$
B:=\left(\begin{array}{ll}
a & b \\
c & d
\end{array}\right):=A(k)
$$

is invertible and not a multiple of the identity. Then, without loss of generality, there is a function $\gamma$ such that for all $x$

$$
A(x)=A(k)-\gamma(x) I
$$

Writing $x \oplus k$ two ways, we find

$$
\frac{k \gamma(x)-(k a+b)}{\gamma(x)-(k c+d)}=\frac{a x+b}{c x+d}
$$

and thus $\gamma$ is a Möbius transformation, say

$$
\gamma(x)=\frac{s x+t}{u x+v}
$$

Since $A(x)$ is invertible if and only if $\gamma(x) \notin S p(B), u B-s I \doteq A(\infty)$ is invertible. Let $C=(v B+t I)(u B-s I)^{-1}$. Then

$$
A(x) \doteq(u x+v) B-(s x+t) I=(C-x I)(u B-s I) .
$$

Note that $A(e) \doteq I$ and thus

$$
C-e I \doteq(u B-s I)^{-1}
$$

Then

$$
\begin{aligned}
(C-x I)(C-y I) & \doteq A(x) A(y)(u B-s I)^{-2} \\
& \doteq A(x \oplus y)(u B-s I)^{-2} \\
& \doteq(C-(x \oplus y) I)(u B-s I)^{-1} \\
& \doteq(C-(x \oplus y) I)(C-e I) .
\end{aligned}
$$


$(e \Rightarrow b)$ : We have two main cases. Suppose first that $A$ is not a multiple of $I$. Let $A$ have trace $T$ and determinant $\Delta$. Then, by the Cayley-Hamilton theorem,

$$
A^{2}=T A-\Delta I
$$

and so

$$
(A-x I)(A-y I)=(T-x-y) A+(x y-\Delta) I .
$$

By (e), if $z:=x \oplus y$, then

$$
(T-z-e) A+(z e-\Delta) I \doteq(T-x-y) A+(x y-\Delta) I
$$

and, since $A$ is not a multiple of $I$,

$$
\frac{z e-\Delta}{z+e-T}=\frac{x y-\Delta}{x+y-T}
$$

Solving for $z(=x \oplus y)$, we get:

$$
x \oplus y=\frac{(T-e) x y-\Delta(x+y)+e \Delta}{x y-e(x+y)+e T-\Delta} .
$$

A direct calculation shows that if $f(x)=\left(x^{2}-T x+\Delta\right) /(x-e)$, then (b) holds for the function $f$.

Now, suppose $A=c I$ for some $c \in \mathbb{R}$. By (e), if $z:=x \oplus y$, then

$$
(c-x)(c-y)=k(c-z)(c-e)
$$

for some $k$. Solving for $z(=x \oplus y)$, we get

$$
x \oplus y=\frac{x y-c(x+y)+c^{2}-k c^{2}+k c e}{k(e-c)} .
$$

A direct calculation shows that if $f(x)=(x-c) /(x-c+k(c-e))$, then (b) holds for the function $f$.

Proof of Theorem 3. Let $y^{\prime \prime}-\alpha y^{\prime}+\beta y=0$ and $T:=-y^{\prime} / y$. If we define $z:=-y^{\prime}$, then

$$
\left\{\begin{array}{l}
z^{\prime}=\alpha z+\beta y \\
y^{\prime}=-z
\end{array}\right.
$$

By the quotient rule, $(z / y)^{\prime}=(z / y)^{2}+\alpha(z / y)+\beta$ and thus

$$
T^{\prime}=p(T)
$$

where $p(x)=x^{2}+\alpha x+\beta$. This is a separable equation and so, if $F:=T^{-1}$, then $F^{\prime}=1 / p$ and $F(T(0))=0$.

Let $f(x)=p(x) /(x-T(0))$ or $p(x)$ according to whether $T(0)$ is finite or not. By Lemma 2,

$$
\frac{\partial}{\partial x} F(x \oplus y)=F^{\prime}(x \oplus y) \frac{\partial}{\partial x}(x \oplus y)=\frac{1}{p(x \oplus y)} \cdot \frac{p(x \oplus y)}{p(x)}=\frac{1}{p(x)}=F^{\prime}(x)
$$


and thus $F(x \oplus y)=F(x)+G(y)$ for some function $G$. By commutativity, $F(x)+$ $G(y)=F(y)+G(x)$ from which it follows that $F(x)-G(x)=F(y)-G(y)$, and so $F$ and $G$ differ by a constant (say $c$ ). Hence,

$$
F(x \oplus y)=F(x)+F(y)+c .
$$

The condition $F(T(0))=0$ ensures that this constant is zero.

\section{ON SINE TYPE ADDITION.}

We say that the binary operation $\diamond$ is of sine-type if it is of the form

$$
u \diamond v=u \varphi(v)+v \varphi(u)
$$

for some function $\varphi$ and is associative.

All examples of sine-type addition arise from secant type addition (Corollary $1)$. As an example, let $f(x)=1 / x$. The secant addition induced by $f$ is normal addition

$$
x \oplus y=x+y
$$

and thus $T(x):=x$ is a homomorphism:

$$
T(x+y)=T(x) \oplus T(y) .
$$

Suppose that there are functions $S$ and $C$ such that $T(x)=S(x) / C(x)$. Then

$$
\frac{S(x+y)}{C(x+y)}=\frac{S(x) C(y)+C(x) S(y)}{C(x) C(y)}
$$

and it is not unreasonable that $S$ and $C$ satisfy the system of functional equations

$$
\left\{\begin{array}{l}
S(x+y)=S(x) C(y)+C(x) S(y) \\
C(x+y)=C(x) C(y) .
\end{array}\right.
$$

This system is solvable: $C(x)=e^{k x}$, and thus $S(x)=x e^{k x}$. Since $u \mapsto(u \ln u) / k$ takes $C$ to $S$, if $\varphi(x)$ is the inverse of $x \ln x$, then

$$
S(x+y)=S(x) \varphi(S(y))+S(y) \varphi(S(x)) .
$$

That is, if

$$
u \diamond v=u \varphi(v)+v \varphi(u)
$$

is the sine-type addition defined by $\varphi$, then

$$
S(x+y)=S(x) \diamond S(y) .
$$

Lemma 4. Let $\diamond$ be the binary operation defined by

$$
x \diamond y=x \varphi(y)+y \varphi(x)
$$


on some interval I containing 0 for some function $\varphi$. Then $\diamond$ is associative if and only if, for some $k \in \mathbb{R}$,

$$
\varphi(x \diamond y)=\varphi(x) \varphi(y)+k x y
$$

Proof. Suppose $\diamond$ is associative. Then

$$
x \varphi(y) \varphi(z)+z \varphi(x \diamond y)=x \diamond y \diamond z-y \varphi(x) \varphi(z)=z \varphi(y) \varphi(x)+x \varphi(z \diamond y)
$$

and therefore

$$
\frac{\varphi(z \diamond y)-\varphi(z) \varphi(y)}{z}=\frac{\varphi(x \diamond y)-\varphi(x) \varphi(y)}{x}
$$

is dependent on $y$ only and so, if we call this quantity $g(y)$, then, solving for $\varphi(x \diamond y)$,

$$
\varphi(x \diamond y)=\varphi(x) \varphi(y)+x g(y) .
$$

By commutativity, $x g(y)=y g(x)$ and so $g(y)=k y$ for some $k$. It follows that

$$
\varphi(x \diamond y)=\varphi(x) \varphi(y)+k x y
$$

Conversely, suppose $\varphi(x \diamond y)=\varphi(x) \varphi(y)+k x y$. Then

$$
(x \diamond y) \diamond z=z \varphi(x) \varphi(y)+k x y z+x \varphi(y) \varphi(z)+y \varphi(x) \varphi(z)
$$

which, since is symmetric with respect to $x$ and $z$, implies associativity.

The equation

$$
\varphi(x \diamond y)=\varphi(x) \varphi(y)+k x y
$$

has been much studied. For $k=0,(8)$ has been studied by Brillouet and Dhombres in [BD]. Sablik [S2] has noted that, at least for $k>0$, the substitution $F(x):=$ $\varphi(x / 2 \sqrt{k})+x / 2$ allows one to rewrite (8) as the 'Baxter equation':

$$
F(x F(y)+y F(x)-x y)=F(x) F(y)
$$

This has been studied by Benz [Be] where it is shown that there are infinitely many discontinuous solutions and where a geometric problem related to (9) is presented. The (unique) continuous solution of (9) has been determined by Volkman and Weigel [VW] and, later, with a shorter proof, by Brzdek [Br].

Proof of Theorem 4. Lemma 4 shows the equivalence of (a) and (b).

$(b \Rightarrow c)$ : Since $(x \diamond y)^{\prime}=\varphi(y)+y \varphi^{\prime}(x)$, differentiating and equating both sides of (b) and then solving for $\varphi^{\prime}(x \diamond y)$ gives (c).

$(c \Rightarrow d)$ : Letting $x=0$ in (c) and using $\varphi^{\prime}(0)=a$ gives $(\mathrm{d})$.

$(d \Rightarrow e)$ : By the definition of $s^{-1}(x), s^{\prime}\left(s^{-1}(x)\right)=\varphi(x)+a x$ and so

$$
\varphi(s(x))=s^{\prime}(x)-a s(x), \quad s(0)=0, \quad s^{\prime}(0)=1 .
$$


Using (d) and (8),

$$
s^{\prime \prime}(x)-a s^{\prime}(x)=\varphi^{\prime}(s(x)) s^{\prime}(x)=a \varphi(s(x))+k s(x)=a\left(s^{\prime}(x)-a s(x)\right)+k s(x)
$$

and (e) follows.

$(e \Rightarrow f)$ : Solving the equation, (f) follows.

$(f \Rightarrow g)$ : Assuming (f), a direct calculation shows

$$
s(x+y)=s(x) s^{\prime}(y)+s(y) s^{\prime}(x)-2 a s(x) s(y) .
$$

Using equation (8), (g) follows.

$(g \Rightarrow h)$ : By definition.

$(h \Rightarrow i)$ : Partial differentiation of both sides of (h) yields

$$
\left(s^{-1}\right)^{\prime}(x \diamond y) \frac{\partial}{\partial x}(x \diamond y)=\left(s^{-1}\right)^{\prime}(x) .
$$

By the integral definition of $s^{-1}$, (i) follows.

$(i \Rightarrow b)$ : By (i)

$$
\varphi(y)+y \varphi^{\prime}(x)=\frac{\varphi(x \diamond y)+a \cdot(x \diamond y)}{\varphi(x)+a x}
$$

which, solving for $\varphi(x \diamond y)$, yields

$$
\varphi(x \diamond y)=\varphi(x) \varphi(y)+y\left[\varphi(x) \varphi^{\prime}(x)+a x \varphi^{\prime}(x)-a \varphi(x)\right] .
$$

By commutativity, (b) follows.

We indicate why Corollary 1 follows from Theorem 4. Note that the equation

$$
\varphi \circ y=y^{\prime}-\frac{1}{2} \alpha y
$$

can be written in integral form:

$$
y^{-1}(x)=\int_{0}^{x} \frac{d t}{\varphi(t)-\frac{1}{2} \alpha t} .
$$

This coincides with the definition of $s$ in Theorem 4 and so Corollary 1 follows by the equivalence of conditions (a) and (e) in Theorem 4.

\section{CONCLUSION.}

We have discussed two types of algebraic addition. In both cases, there exists a 'homomorphism'. For example, sine-type addition with $\varphi(x):=\sqrt{1-x^{2}}$ satisfies

$$
\sin (x+y)=\sin (x) \diamond \sin (y)
$$

and secant addition with $f(x):=\left(1-x^{2}\right) / x$ satisfies

$$
\tanh (x+y)=\tanh (x) \oplus \tanh (y) .
$$


In general, there exists $F$ such that

$$
F(x+y)=F(x) * F(y)
$$

Furthermore, the functions $F$ are invertible and, for fixed $c$,

$$
F^{-1}(x * c)=F^{-1}(x)+F^{-1}(c) .
$$

If $g(x):=F^{-1}(x) / F^{-1}(c)$ and $m(x):=x * c$, then $g$ and $m$ satisfy 'Abel's equation'

$$
g(m(x))=g(x)+1 .
$$

This allows a closed formula for iterates of $m$ and, at the same time, a definition of "fractional iterates" of $m$ :

$$
m_{t}(x):=g^{-1}(g(x)+t) \text { for } t \geq 0 .
$$

In our cases, $g$ is differentiable and if $p(x):=1 / g^{\prime}(x)$, then

$$
\frac{\partial}{\partial x}(x * y)=\frac{p(x * y)}{p(x)} .
$$

secant addition is characterized by $p(x)$ being a polynomial of degree at most 2 , (e.g. $p(x)=1-x^{2}$ in the case with $F(x)=\tanh x$ ) whereas sine-type addition is characterized by another class of functions (e.g., $p(x)=\sqrt{1-x^{2}}$ in the case with $F(x)=\sin x)$. Other choices of $p(x)$ give rise to algebraic addition:

$$
\int_{a}^{x} \frac{d t}{p(t)}+\int_{a}^{y} \frac{d t}{p(t)}=\int_{a}^{x * y} \frac{d t}{p(t)}
$$

An interesting example is when $p(x):=\sqrt{\left(1-x^{2}\right)\left(1-k^{2} x^{2}\right)}$ for a fixed $k \in$ $(0,1)$ (the cases when $k=0$ and $k=1$ are covered by the two examples above). The addition defined by this is

$$
x * y=\frac{x p(y)+y p(x)}{1-k^{2} x^{2} y^{2}}
$$

and has been well studied; for example, the Jacobi elliptic function $\operatorname{sn}(x, k)$ is a homomorphism $([\mathrm{WW}])$.

\section{REFERENCES}

[A1] J. Aczel, "Lectures on Functional Equations and their Applications", Academic Press, New York, 1966.

[A2] J. Aczel, The state of the second part of Hilbert's fifth problem, Bull. Amer. Math. Soc. (N.S.) 20 (1989), no. 2, 153-163.

[A3] J. Aczel, A Mean Value Property of the Derivative of Quadratic Polynomials - without Mean Values and Derivatives, Mathematics Magazine, Vol 58, no. 1 (1985) 42-45.

[Au] G. Aumann, Problem \#21 for Ninth International Symposium on Functional Equations (1971), 
http : //riesz.math.klte.hu/isfe//isfe1971_9/info.php

[Be] W. Benz Ein Beltrag zu einem Problem von Hernn Fenyo Abh. Math. Sem. Univ. Hamburg 57 (1987) 21-25.

[Bo] R. Bojanic, Une propriete caracteristique des courbes du second degre Bull Soc. Math. Phys. Serbie, 1, (1949). no. 3-4, 105-111.

[Br] J. Brzdek, On the Baxter functional equation, Aequationes Math. 52 (1996), no. 1-2, 105-111.

[BB] J.M. Borwein and P.B. Borwein, "Pi and the AGM", Wiley, New York, 1987.

[BD] N. Brillouet and J. Dhombres Equations fonctionnelles et recherche de sousgroupes, Aequationes Math. 31 (1986), no. 2-3, 253-293.

[CMc] R. Coleman and F. McGuinness, Rational Formal Group Laws, Pacific J. Math. 147 (1991), no. 1, 25-27.

[K] V.S. Kalnitsky, Means Generating the Cone Sections and the Third Degree Polynomials, SPb. Math. Society Preprint 2004-04.

[N1] S. Northshield, On iterates of Möbius transformations on fields, Math. Comp. 70 \#235 (2000) 1305-1310.

[N2] S. Northshield, Associativity of the Secant Method, American Math. Monthly 109 (2002), no. 3, 246-257.

[S1] M. Sablik, Final part of the answer to Hilbert's question Functional equations - results and advances, 231-242, Adv. Math. (Dordr.), 3, Kluwer Acad. Publ., Dordrecht, 2002.

[S2] M. Sablik, On some local topological semigroups, Aequationes Math. 44 (1992), no. 2-3, 194-219.

[ST] J.H. Silverman and J. Tate, "Rational Points on Elliptic Curves", Springer, New York, 1992.

[VW] P. Volkmann and H. Weigel, Uber ein Problem von Fenyo, Aequationes Math. 27 (1984), no. 1-2, 135-149.

[WW] E.T. Whittaker and G.N. Watson, "A Course of Modern Analysis", Fourth Edition, Cambridge Univ. Press, 1952.

E-mail address : samuel.northshield@plattsburgh.edu 